\title{
Answer to the comments on "Confirming geomagnetic Sfe by means of a solar flare detector based on GNSS"
}

\author{
Juan José Curto ${ }^{1, *}$, Jose Miguel Juan², and Cristhian Camilo Timoté ${ }^{2}$ \\ ${ }^{1}$ Observatorio del Ebro, (OE) CSIC - Universitat Ramon Llull (URL), 43520 Roquetes, Spain \\ ${ }^{2}$ Research group on Astronomy and Geomatics (gAGE), Universitat Politècnica de Catalunya (UPC), Jordi Girona, 1-3, 08034 Barcelona, \\ Spain
}

Received 7 February 2020 / Accepted 11 March 2020

\begin{abstract}
Hernández-Pajares and García-Rigo have written a document criticizing our paper "Confirming geomagnetic Sfe by means of a solar flare detector based on GNSS. J Space Weather Space Clim 9: A42. https://doi.org/10.1051/swsc/2019040" (Curto et al., 2019). The main goal of our paper was to define a methodology based on GNSS measurements that is able to detect solar flares (SF) in an automatic way. This methodology was used to confirm Sfe (SF effects) detected by geomagnetism in an unsupervised manner. In their document, Hernández-Pajares and García-Rigo posed two objections related to the correctness and the novelty of the methodology used in our paper. This document is a reply to these objections and concludes that they are not relevant.
\end{abstract}

Keywords: Sfe detection / GNSS-SF / rise time / solar flares / ionospheric disturbances

\section{Introduction}

The aim of the work presented in Curto et al. (2019) was to define a methodology based on GNSS measurements that is able to detect solar flares (SF) in an automatic way, to confirm solar flare effects (Sfe) detected by geomagnetism in an unsupervised manner.

The basic measurements used to define the SF detector presented in Curto et al. (2019) are the slant total electron content (STEC) variations, corrected by an obliquity factor (see below). This obliquity factor was introduced to reduce the increase in STEC variations at low elevations, i.e. to normalise such STEC variations.

Based on these corrected STEC values, denoted as $\triangle \mathrm{STEC}_{i}^{j}$, we analysed three candidates for this automatic detector during an entire solar cycle (see Curto et al., 2019 for more information):

1. $\triangle \mathrm{STEC}$ at the subsolar point (SSP).

2. $\Delta^{2}$ STEC at the subsolar point (SSP).

3. Correlation coefficient, $\rho$, of the $\Delta^{2}$ STEC fitting.

Our work concluded that the combined use of the $\Delta^{2}$ STEC and $\rho$ detectors provides a successful ratio of Sfe confirmations.

\footnotetext{
*Corresponding author: jjcurto@obsebre.es
}

The comments received by Hernández-Pajares and GarcíaRigo (hereafter HP\&GR) regarding our paper can be summarized as follows:

1. Mistakes in the methodology could invalidate our results.

2. The SF detectors used in our paper were already defined in their papers.

The present document aims to refute these objections.

\section{Regarding the claim about mistakes in the methodology}

It is clear that HP\&GR assume a single-layer model for the ionosphere, where the obliquity factor is a factor that converts the vertical total electron content (VTEC) to the slant total electron content (STEC) in such a way that, for a satellite " $j$ " and a receiver " $i$ ", the ionospheric delay can be written as:

$$
\operatorname{STEC}_{i}^{j}=m(\varepsilon) \cdot \operatorname{VTEC}(A)
$$

where $A$ is the ionospheric pierce point (IPP) (i.e. the intersection of the line of sight vector with the ionospheric layer) and $m(\varepsilon)$ is the so-called obliquity factor or mapping function, defined as: 

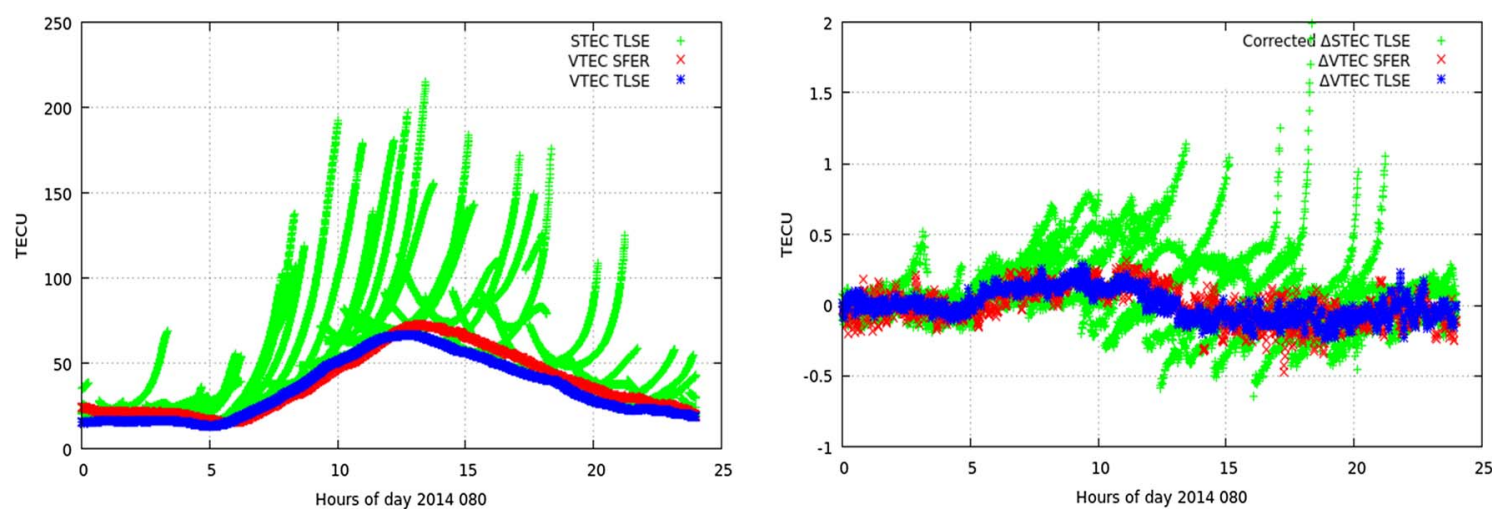

Fig. 1. Left panel: STEC values from the receiver TLSE (green), VTEC at TLSE (blue) and VTEC at SFER (red). Right panel: corrected variations of the STECs in the left panel (green) and variations of VTEC (blue and red).

$$
m(\varepsilon)=\sqrt{\frac{1}{1-\left(\frac{R_{\mathrm{E}} \cdot \cos (\varepsilon)}{R_{\mathrm{E}}+h_{\mathrm{ion}}}\right)^{2}}}
$$

where $R_{\mathrm{E}}$ is the Earth radius, $\varepsilon$ is the satellite elevation angle, and $h_{\text {ion }}$ is the height of the ionospheric layer.

In GNSS, one of the basic ionospheric measurements for extracting STEC in (1) is the well-known geometry-free combination of carrier-phase $\left(L_{\mathrm{GF}}\right)$, that shall be corrected from geometric effects such as antenna phase centres (of both satellite and receiver) and from the satellite wind-up. $L_{\mathrm{GF}}$ is related to the STEC by:

$$
L_{\mathrm{GF}_{i}}^{j}=\mathrm{STEC}_{i}^{j}+B_{\mathrm{GF}_{i}^{j}}^{j}
$$

where $B_{\mathrm{GF}_{i}}^{j}$ is a constant per arc, which includes the so-called carrier phase ambiguities and instrumental biases (of both the satellite and the receiver).

If $B_{\mathrm{GF}_{i}}^{j}$ is known, thence VTEC can be obtained from the $L_{\mathrm{GF}}$ measurements through:

$$
\operatorname{VTEC}(\mathrm{A})=\frac{1}{m(\varepsilon)}\left(L_{\mathrm{GF}^{j}}^{j}-B_{\mathrm{GF}_{i}^{j}}^{j}\right)
$$

Therefore, using the previous relationship one could relate an increase of VTEC at $A$ with the occurrence of a SF.

However, the knowledge of $B_{\mathrm{GF}_{i}}^{j}$ is not trivial. Therefore, it is common to cancel this term by taking differences on time of $L_{\mathrm{GF} i}^{j}$ along a continuous arc of data, where it is assumed that $B_{\mathrm{GF}_{i}}^{j}$ is constant. Thus, this differences on time can be identified with differences of $\mathrm{STEC}_{i}^{j}$ :

$$
L_{\mathrm{GF}_{i}^{j}}^{j}\left(t_{B}\right)-L_{\mathrm{GF}_{i}^{j}}^{j}\left(t_{A}\right)=\operatorname{STEC}_{i}^{j}\left(t_{B}\right)-\operatorname{STEC}_{i}^{j}\left(t_{A}\right)
$$

where $A$ and $B$ are the IPPs at the times $t_{A}$ and $t_{B}$, respectively.

Applying (1), the previous relationship can be written as:

$$
L_{\mathrm{GF}_{i}^{j}}^{j}\left(t_{B}\right)-L_{\mathrm{GF}_{i}^{j}}^{j}\left(t_{A}\right)=m\left(\varepsilon_{B}\right) \cdot \operatorname{VTEC}(B)-m\left(\varepsilon_{A}\right) \cdot \operatorname{VTEC}(A) .
$$

Following the HP\&GR reasoning this last expression could be approximated as:

$$
L_{\mathrm{GF}_{i}^{j}}^{j}\left(t_{B}\right)-L_{\mathrm{GF}_{i}^{j}}^{j}\left(t_{A}\right) \approx m\left(\varepsilon_{A}\right) \cdot \Delta \operatorname{VTEC}(A)
$$

Therefore, the increase of VTEC at the IPP can be approximated by:

$$
\Delta \operatorname{VTEC}(A) \approx \frac{1}{m\left(\varepsilon_{A}\right)}\left(L_{\mathrm{GF}_{i}^{j}}^{j}\left(t_{B}\right)-L_{\mathrm{GF}_{i}^{j}}^{j}\left(t_{A}\right)\right) .
$$

This is the expression that HP\&GR are thinking on their first comment.

However, interpreting the right side of equation (2) as a VTEC variation is just an approximation that assumes that the STEC variations are linked to an increase of the VTEC at the IPP $A$. This assumption would be only true for a geostationary satellite, where both, $A$ and $B$, are the same IPP. Nevertheless, in general, STEC variations depends also on the spatial gradients which, at low elevations, use to be, by far, the predominant component of the STEC variations.

In order to illustrate the dependency of the STEC variations on the spatial gradient, Figure 1 depicts, in the left panel, the STEC values for the receiver TLSE (South of France) during the day 080 in 2014. These STEC values, in green, are obtained after solving, for each arc, the constant $B_{\mathrm{GF} i}^{J}$. The solution for these constants are computed by means of a sophisticated process that includes worldwide carrier phase ambiguity fixing (see Rovira-Garcia et al., 2016 for more details). Beside these very accurate STEC values, the VTEC values for TLSE and SFER (South of Spain) are also depicted in the left panel. The VTEC values are obtained from these very accurate STECs and using a dual layer ionospheric model that, as it is shown in Rovira-Garcia et al. (2016) or in Rovira-Garcia et al. (2019), is several times more accurate than the standard single layer ionospheric models. In the right panel, the difference of these three magnitudes along 1 minute are depicted. As it can be seen, while the VTEC variations (computed from the ionospheric model) are less than 0.2 TECU $\left(1\right.$ TECU $=10^{16}$ electrons $\left./ \mathrm{m}^{2}\right)$, the STEC variations (computed directly using Eq. (2)) can be larger than 1 TECU (even if one puts and elevation mask of 20 degrees). These larger variations of the STECs cannot be attributed to the possible low latitude of the IPPs (this is the reason we depict also the VTEC for SFER). Thus, the variations in the right side of equation (2) are far from being a VTEC variation.

Therefore, in our paper, we interpreted the right side of equation (2) as it is: a STEC variation multiplied by an obliquity 

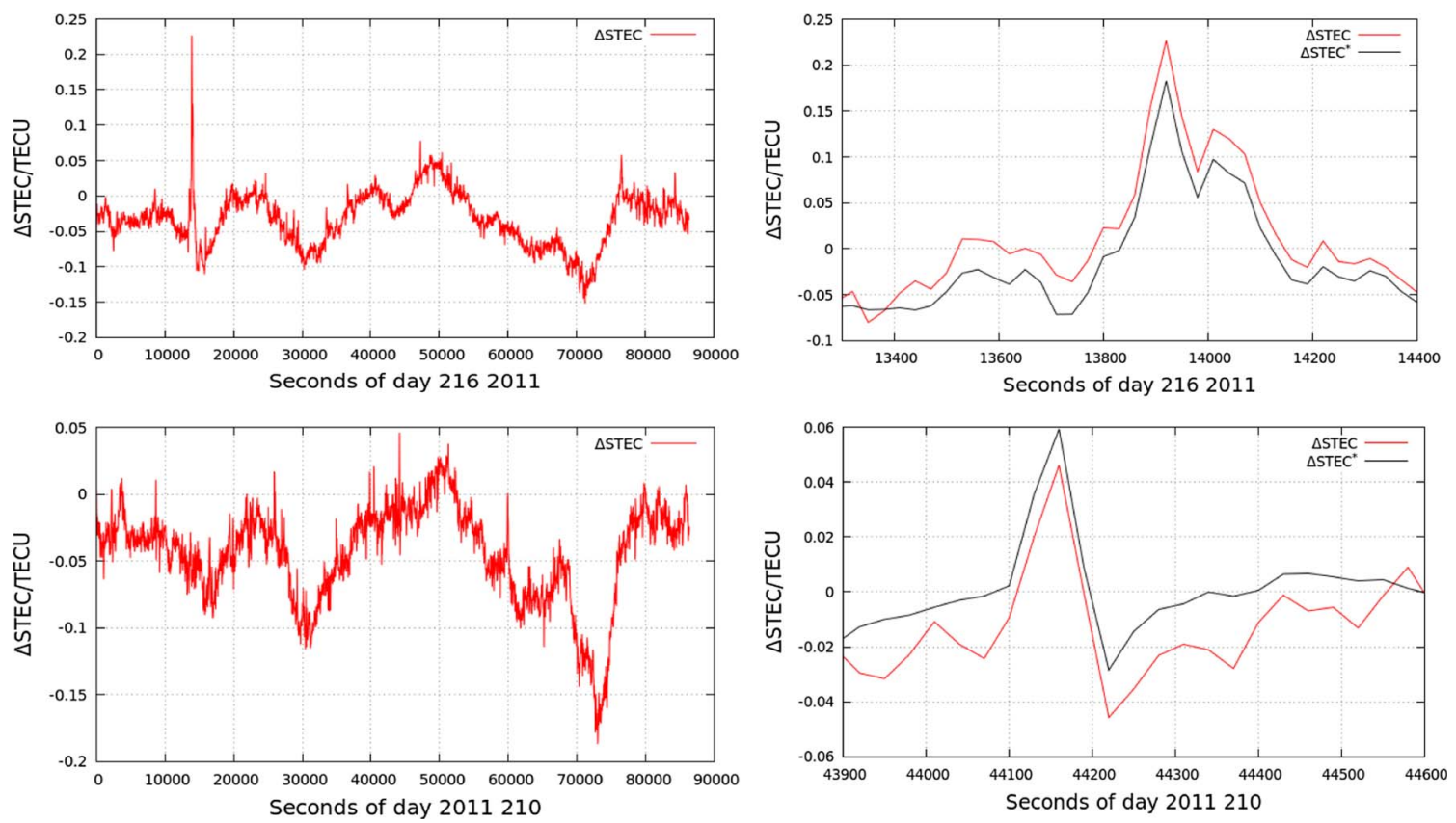

Fig. 2. Left column: $\triangle$ STEC at the SSP during two days of 2011: day 216 at the top and day 210 at the bottom. Right column: in red, an enlarged view of the plots on the left side during the same time interval as in Hernández-Pajares et al. (2012). Additionally, $\Delta$ STEC* is depicted in black.

factor $\left(M\left(\varepsilon_{A}\right)=\frac{1}{m\left(\varepsilon_{A}\right)}\right)$ which mitigates the large variations of STEC at low elevations. Thus, we define $\triangle \mathrm{STEC}_{i}^{j}$ as this corrected (normalised) STEC variation:

$$
\Delta \mathrm{STEC}_{i}^{j}=M\left(\varepsilon_{A}\right) \cdot\left(L_{\mathrm{GF}_{i}^{j}}^{j}\left(t_{B}\right)-L_{\mathrm{GF}_{i}^{j}}^{j}\left(t_{A}\right)\right) .
$$

This is the definition done in Curto et al. (2019).

In our opinion this is a more general interpretation than considering this as a VTEC variation at a specific IPP. This different interpretation (in fact, just a different name) for the same measurement is the origin of the two supposed mistakes that HP\&GR claim in their comments:

First mistake: "The difference of ionospheric carrier phases in the same phase-continuous transmitter-receiver arc, provides directly the STEC variation, without the need of any mapping function term".

Answer: In our paper, we clearly defined $\triangle \mathrm{STEC}_{i}^{j}$ as the STEC variations corrected by the obliquity factor. As we have explained, considering $\triangle \mathrm{STEC}_{i}^{j}$ as a VTEC variation at a given IPP is just a rough approach.

Second mistake: "In GSFLAI the linear dependence is in terms of the Vertical TEC variation, not the Slant TEC one".

Answer: This could have happened if we had defined $\triangle \mathrm{STEC}_{i}^{j}$ without correcting from the obliquity factor, but this not the case.

To sum up, there is no mistakes in our strategy and the disagreement regarding HP\&GR is in how the STEC variations corrected by the obliquity factor are named/interpreted. In this point, we think that our interpretation is more adequate.
HP\&GR also state that, due to these supposed mistakes, our results and conclusions could be erroneous. After having processed data from an entire solar cycle, we have been able to satisfactorily cross-check our results with those found in the literature: in our opinion, this task is mandatory in any scientific work. For instance, Figure 2 depicts two of the examples that can be found in Hernández-Pajares et al. (2012) and correspond to days 216 and 210 of 2011 (during those days, two weak SF occurred, as reported in Hernández-Pajares et al., 2012). Using the same time intervals as in Hernández-Pajares et al. (2012), the panels on the right side show that our $\triangle$ STEC detector is able to reproduce similar results to those in Hernández-Pajares et al. (2012). Indeed, the amplitude of the peak reaches to 0.2 TECU. Additionally, the detection is better if we use $\triangle$ STEC $^{*}$ (a parameter also defined in our paper) because the noise is reduced and the detection is clearer. However, this detection can be done only if short time intervals are exclusively considered, as it has been done in the panels on the right side of Figure 1. In contrast, if the time intervals are expanded to an entire day (as in the left panels), it can be observed that in some instants, the values of $\triangle$ STEC can be at the same level as or even larger than the values during the SFs. As shown in our paper, these results indicate the difficulty in establishing thresholds for unsupervised SF detections using this detector.

\section{Regarding the claim about the novelty of the SF detectors}

As mentioned in the introduction of the present answer, the goal of Curto et al. (2019), regarding the GNSS SF detectors, was not to present three new SF detectors but to analyse their 
suitability for the automatic detection of SF. In this sense, the key results pertaining to this goal are the three complementary cumulative distribution functions presented in the three panels in Figure 9 in Curto et al. (2019). Indeed, from Figure 9, our conclusion was that $\triangle \mathrm{STEC}$ is not adequate for the automatic detection of SF. On the contrary, the two other detectors are more suitable for this automatic task.

We have not presented any of these SF detectors as a "new Solar Flare index", as HP\&GR state in their comments. The only sentence that could be interpreted in this sense can be found at the end of Section 2.2: Data and Thresholding:

"In conclusion, the value of $\rho$ can be used as a measure of the confidence level for SF detection. This is a novelty with respect to previous SF detectors based on GNSS measurements, because it represents a self-consistent way for providing confidence to the SF detections".

When we compared $\triangle$ STEC with the GNSS solar flare activity indicator (GSFLAI), we stated that they are "similar" (line 174) or "equivalent" (line 190) because, over the same events, the results obtained using our $\triangle$ STEC detector are very similar, but not exactly the same, to those presented in HernándezPajares et al. (2012) (see, for instance, the aforementioned Fig. 2). There are several reasons that could explain these differences, some of which are the use of the slope (used in Hernández-Pajares et al., 2012) instead of $\triangle$ STEC, the cadence of the data ( $1 \mathrm{~s}$ and $30 \mathrm{~s}$ ) or the outlier exclusion strategies.

Moreover, note that the detector consists of more than just a model relating the angle between the IPP and solar zenith $(\chi)$ with each of $\triangle \mathrm{STEC}_{i}^{j}$. For instance, Wan et al. (2005) proposed a proportional relationship of the sudden increase in total electron content (SITEC) with $\cos \chi^{1}$, while Hernández-Pajares et al. (2012) defined GSFLAI by means of a linear relationship between $\triangle \mathrm{STEC}_{i}^{j}$ and $\cos \chi$. The detector, i.e., the parameters of the relationship, also depends on how these parameters are estimated: Kalman filter or least squares, constraint/smooth equations, etc. In our case, a linear relationship was assumed (as GSFLAI) instead of a proportional one (as SITEC). However, we realized that dropping the independent term of this linear relationship, the results were still worse than those presented in the left panel of Figure 9 in our paper. Therefore, we decided to constrain the value of the independent term to zero in such a way that our detector, $\triangle$ STEC, can be close to the GSFLAI or SITEC depending on the constraint imposed on the independent term. For instance, using the case presented in the top-right panel of Figure 1 in this document, Figure 3 presents the $\triangle$ STEC using different constraints on the independent term: a moderate constraint, as it is used in $\triangle$ STEC (in red), a heavy constraint (in green), which should be equivalent to the SITEC in Wan et al. (2005), and no constraint on the independent term (in blue), which should be equivalent to the GSFLAI (assuming that the GSFLAI drops the independent term and considering the G2 definition for the GSFLAI that HP\&GR mention in their document).

\footnotetext{
${ }^{1}$ Actually, the relationship shown in the paper is the inverse of the Chapman function, but, as the authors state in their paper, it can be approximated as a relationship with $\cos \chi$.
}

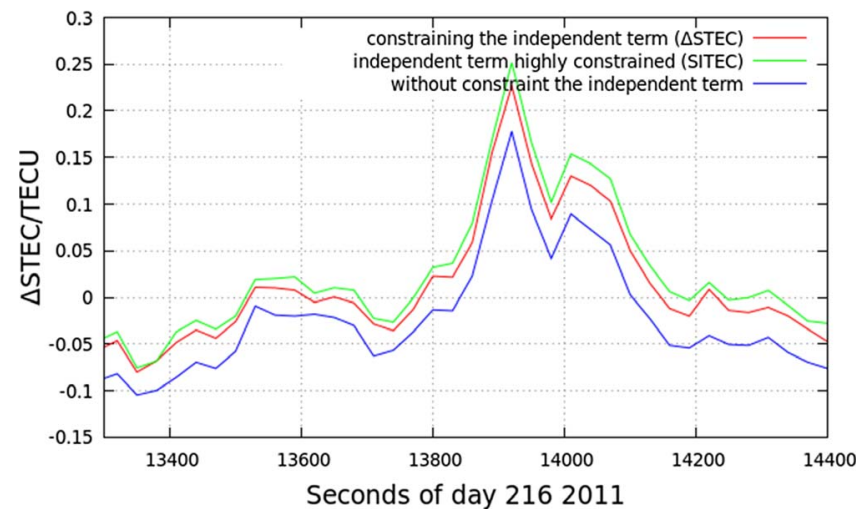

Fig. 3. $\triangle$ STEC computed using three different constraints on the independent term of the linear fitting: without constraint (in blue), hard constraint (in green) and moderate constraint (in red).

As shown, a moderate constraint on the independent term causes $\triangle$ STEC to behave within the GSFLAI and SITEC outputs. In this way, in this example, $\triangle \mathrm{STEC}$ is closer to the SITEC than to the GSFLAI.

In summary, there are some aspects of the implementation of the SF detectors that are not explicitly shown in the corresponding articles that describe the detector but that could affect their performance. This is the reason why we have compared only the results, concluding that they are similar, and for the same reason, we limited our conclusion to the poor performance of $\triangle \mathrm{STEC}$ as an automatic detector. However, in our opinion, this conclusion should be extended to detectors based on the sudden increase in the STEC at the SSP (not only $\triangle$ STEC or the GSFLAI but others defined in previous works, such as the case of the SITEC). Consequently, coming back to the novelty, it would be a non-sense to present $\triangle$ STEC as a new automatic detector (as HP\&GR are claiming) and, after that, to conclude that it does not work as an automatic detector. Therefore, this is an irrelevant discussion because, in our paper, we are not using $\triangle$ STEC or the GSFLAI for automatic detections.

Finally, HP\&GR state that, in previous works, they have used the second difference in time of the VTEC at the SSP as an indicator of solar activity. Following the same line of thought as HP\&GR, one could state that the first difference in time of the VTEC at the SSP was used in several works prior to Hernández-Pajares et al. (2012). Therefore, one should conclude that there is no novelty in the GSFLAI definition. However, we think that this is not the case and that, as we have commented before, in SF detection, it is important not only to determine which physical property should be used to characterize the SF occurrence but also to select the methodology necessary to obtain the corresponding parameters. Moreover, as we stated before, in our work, we did not claim that the detectors were novel, but simply discussed the best way to use them as automatic SF detectors, which, in the end, should be their goal.

For instance, HP\&GR refer to Monte-Moreno \& HernándezPajares (2014), where they used the "subsolar Vertical Total Electron Content double difference in time" as an indicator of solar activity. In fact, they used in this paper the difference in time (rate) of the GSFLAI (GSFLAIR) which, in our case, 

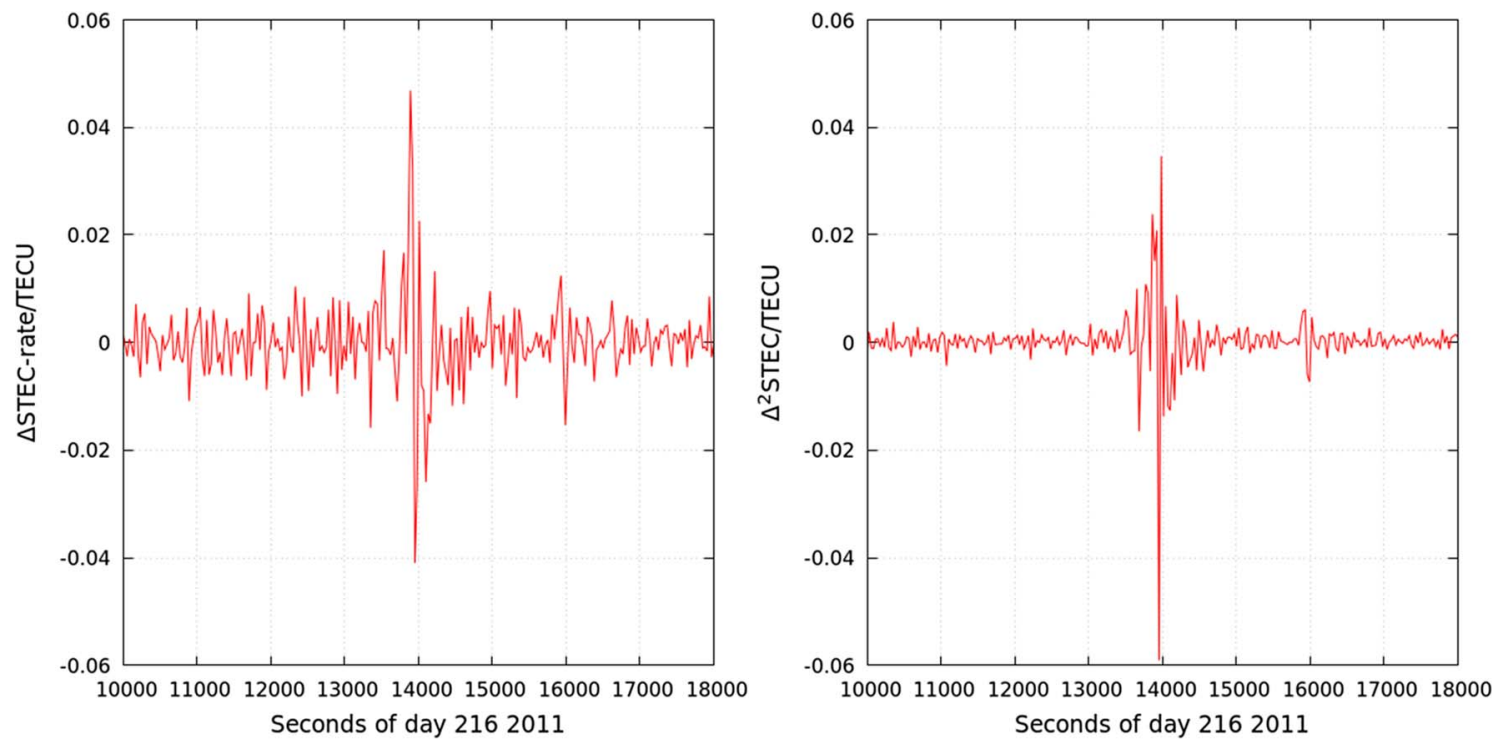

Fig. 4. Left panel: $\triangle \mathrm{STEC}$ rate for the example depicted Figure 3. Right panel: $\Delta^{2}$ STEC for the example depicted in Figure 3.

should be similar to the rate of $\triangle \mathrm{STEC}$. However, $\Delta^{2}$ STEC is not equivalent to the $\triangle$ STEC rate. Indeed, the first one is obtained by fitting a linear model to the satellite-receiver $\Delta^{2}$ STEC $_{i}^{j}$, while the second one would be obtained by differencing in time $\Delta$ STEC at the SSP. In this way, $\Delta^{2}$ STEC should be the rate of $\triangle$ STEC* defined in our paper. In order to see the differences, Figure 4 depicts for the same example in Figure 3 the $\triangle$ STEC rate in the left panel, and the $\Delta^{2}$ STEC in the right panel. It can be seen that both detectors have similar peaks when the SF occurs. However, the noise level of the $\triangle$ STEC rate is several times larger than the noise in the $\Delta^{2}$ STEC detector. Probably, this is the reason why Monte-Moreno \& HernándezPajares (2014) set a threshold of 0.025 TECU for the SF detection based on the "experience of the authors comparing with other sources". In our case, we set a threshold for $\Delta^{2}$ STEC to just 0.01 TECU based in our statistic results, which are shown in Figure 9 of our paper (middle panel). As it can be seen in this panel we are able to detect, in a confident way, much more SFs than putting the threshold in 0.025 TECU.

We have to recognize that we did not aware the work in Monte-Moreno \& Hernández-Pajares (2014), otherwise we would remark in our work this improvement of $\Delta^{2}$ STEC with respect to GSFLAIR.

\section{References}

Curto JJ, Juan JM, Timoté CC. 2019. Confirming geomagnetic Sfe by means of a solar flare detector based on GNSS. J Space Weather Space Clim 9: A42. https://doi.org/10.1051/swsc/2019040.

Hernández-Pajares M, García-Rigo A, Juan JM, Sanz J, Monte E, Aragón-Àngel A. 2012. GNSS measurement of EUV photons flux rate during strong and mid solar flares. Space Weather 10(12): doi: https://doi.org/10.1029/2012SW000826.

Monte-Moreno E, Hernández-Pajares M. 2014. Occurrence of solar flares viewed with GPS: Statistics and fractal nature. J Geophys Res Space Phys 119(11): 9216-9227. https://doi.org/10.1002/ 2014JA020206.

Rovira-Garcia A, Juan JM, Sanz J, González-Casado G, Ibáñez D. 2016. Accuracy of ionospheric models used in GNSS and SBAS: methodology and analysis. J Geod 90(3): 229-240. https://doi.org/ 10.1007/s00190-015-0868-3.

Rovira-Garcia A, Ibáñez-Segura D, Orús-Perez R, Juan JM, Sanz J, González-Casado G. 2019. Assessing the quality of ionospheric models through GNSS positioning error: methodology and results. GPS Solu 24(1): 4. https://doi.org/10.1007/s10291-019-0918-z.

Wan W, Liu L, Yuan H, et al. 2005. The GPS measured SITEC caused by the very intense solar flare on July 14, 2000. Adv Space Res 36: 2465-2469. https://doi.org/10.1016/j.asr.2004.01.027.

Cite this article as: Curto JJ, Juan JM \& Timoté CC 2020. Answer to the comments on "Confirming geomagnetic Sfe by means of a solar flare detector based on GNSS". J. Space Weather Space Clim. 10, 16. 